\title{
TSpace
}

The University of Toronto's research repository

\section{Accepted Manuscript of Pivotal Advance: Phospholipids determine net membrane surface charge resulting in differential localization of active Rac1 and Rac2}

\section{How to cite TSpace items}

Always cite the published version, so the author(s) will receive recognition through services that track citation counts, e.g. Scopus. If you need to cite the page number of the TSpace version ${ }^{1}$ because you cannot access the published version ${ }^{2}$, then cite the Tspace version in addition to the published version.

Published version citation:

Magalhaes MA, Glogauer M. Pivotal Advance: Phospholipids determine net membrane surface charge resulting in differential localization of active Rac1 and Rac2. J Leukoc Biol. 2010 Apr;87(4):545-55.

TSpace version citation:

Magalhaes MA, Glogauer M. Pivotal Advance: Phospholipids determine net membrane surface charge resulting in differential localization of active Rac1 and Rac2. TSpace. Available at http://hdl.handle.net/XXXX/XXXXX. Replace the 'XXXX/XXXXX' with the item handle from the URL, i.e. the last 9 digits.

Uses of this material require specific permission from the publisher.

\footnotetext{
${ }_{1}^{1}$ TSpace version: includes the pre-print/original manuscript (version before peer review) and post-print/ accepted manuscript (version after peer-review and editing).

${ }^{2}$ Published version: the publisher's final PDF.
} 


\section{Phospholipids determine net membrane surface charge resulting in differential localization of active Rac1 and Rac2}

Running title: Membrane phospholipids regulate Rac localization

Marco A. O. Magalhaes ${ }^{1}$ and Michael Glogauer ${ }^{2 *}$

1 Department of Anatomy and Structural Biology, Albert Einstein College of Medicine, Yeshiva University, Bronx, NY, 10461.

2 CIHR Group in Matrix Dynamics and Dental Research Institute, University of Toronto, 2Faculty of Dentistry, Toronto, Ontario, Canada M5G 1G6

* Corresponding author

241 Fitzgerald Building

150 College Street

University of Toronto, Toronto, Canada M5S 3E2, Phone: (416) 978- 6685

E-mail: michael.glogauer@utoronto.ca

Keywords: Neutrophils, Phagocytosis, Chemotaxis, Rac, Charge

Summary sentence: This manuscript provides evidence that neutrophils regulate membrane lipid composition and membrane surface charge to localize signaling pathways through the recruitment of differentially charged proteins.

Total character count: 28878

Color figures: 5

References: 46

Words in abstract: 210

Summary sentence: 25 


\section{Abstract}

In this investigation, we used primary murine neutrophils to demonstrate that local changes in membrane phospholipid composition alter the net cytoplasmic membrane surface charge, which results in selective recruitment of either Rac1 or Rac2 based on the net charge of their respective C-terminal domains. Murine neutrophils undergoing either chemotaxis or carrying out phagocytosis were transfected with Kras4B derived membrane charge biosensors and lipid markers which allowed us to simultaneously monitor the levels of $\mathrm{PIP}_{2}, \mathrm{PIP}_{3}$ and PS and net membrane charge at the newly developing phagosome membrane and during chemotaxis. Our results indicate that the combination of $\mathrm{PIP}_{2}, \mathrm{PIP}_{3}$ and PS generate a high negative charge (-8) at the plasma membrane of actin rich pseudopods, where active Rac1 preferentially localizes during phagosome formation. The lipid metabolism that occurs during phagosome maturation results in the localized depletion of $\mathrm{PIP}_{2}$, and $\mathrm{PIP}_{3}$ in the phagosome membrane and creates a moderately negative net charge that correlates with the localization of active Rac2. Conversely, the accumulation of $\mathrm{PIP}_{3}$ at the leading edge membrane during chemotaxis generates a polarized accumulation of negative charges that recruits Rac1. These results provide evidence that neutrophils may regulate membrane lipid composition and membrane surface charge in order to localize signaling pathways through the recruitment of differentially charged proteins. 


\section{Introduction}

In addition to the compelling evidence demonstrating the role of phospholipids as signaling molecules, the negative charges carried by these lipids are known to recruit positively charged proteins to localized membrane domains. The contribution of phospholipids to the negative charge of cellular membranes was recently reviewed by Yeung and Grinstein [1]. Briefly, monovalent lipids including phosphatidylserine (PS) and polyvalent phospholipids including phosphatidylinositol-4,5-bisphosphate $\left(\mathrm{PIP}_{2}\right)$ and phosphatidylinositol-3,4,5-triphosphate $\left(\mathrm{PIP}_{3}\right)$ are asymmetrically distributed at the cytosolic surface of membranes and confer a negative charge to the plasma membrane, creating an electric field equivalent to $10^{5} \mathrm{~V} / \mathrm{cm}$ [2-5]. It is well established that electrostatic interactions between positively charged molecules and negatively charged phospholipids are important for targeting signaling pathways $[1,6]$. As proposed by McLaughlin, these interactions may be modulated by the charges in either the membrane or the protein [7]. The role of the electrostatic interactions in the recruitment of positively charged proteins to the plasma membrane has been analyzed in several cell lines but previous studies designed to analyze phospholipid localization and metabolism in primary neutrophils are lacking, since these critical white blood cells are short-lived and terminally differentiated. The recent development of a transient transfection technique for primary murine neutrophils has allowed us to study lipid metabolism and membrane charge in real time in these important infection-fighting cells $[8,9]$. We describe here experiments which demonstrate that local changes in membrane phospholipid composition alter the net membrane charge which impacts Rac isoform localization based on the C-terminal domain of these small GTPases. 
Rac1 and Rac2 carry a positive charge at the C-terminus, where Rac1 has an estimated +7 charge conferred by six basic residues (KKRKRK) at the PB domain while Rac2 has an estimated +4 charge conferred by three basic residues (RQQKRA). Recent studies have demonstrated that the polybasic sequence (PB) of Rac1 is important for protein localization and function, including the regulation of NADPH oxidase assembly [10, 11], Rac1 binding to $\mathrm{PIP}_{3}[12]$, Rac1 membrane targeting $[13,14]$ and nuclear import of Rac1 [15-17]. Yeung et al (2006) [18] demonstrated that Rac1 and K-Ras localize to plasma membrane compartments based on their PB domain net charge [19] but the role of membrane charge in the localization of moderately charged proteins including Rac2 still needs to be elucidated. Considering the differences in the PB domains and the different roles of Rac isoforms in neutrophil functions [14, 20, 21], we wanted to analyze the lipid-dependent charge distribution in primary neutrophils and the charge-dependent selective recruitment of Rac isoforms during phagocytosis and chemotaxis. Our results presented here indicate that the combination of PS, $\mathrm{PIP}_{2}$ and $\mathrm{PIP}_{3}$ generate a high negative charge (-8) at the plasma membrane of actin rich pseudopods during phagocytosis and chemotaxis, which preferentially recruits Rac1. The lipid metabolism that follows receptor activation during phagocytosis results in the depletion of $\mathrm{PIP}_{2}, \mathrm{PIP}_{3}$ and PS at the phagosome membrane and creates a moderate negative net charge that recruits Rac2 at the expense of Rac1. We show here that lipid metabolism at membranes regulates the net membrane charge which is responsible for selective recruitment of charged proteins including positively charged small GTPases based on their own inherent net positive charge. 


\section{Materials and Methods}

DNA constructs. The Kras4B-derived charge probes were a kind gift from Dr. John E. Silvius (McGill University). Briefly, chimeric proteins were engineered by fusing the carboxy-terminal sequence of K-ras4B to enhanced green fluorescent protein (EGFP). Mutations were introduced into the K-ras4B-derived portion of this sequence by oligonucleotide-directed mutagenesis, creating an array of charge biosensors ranging from +2 to $+8[22]$. The constructs use were EK (SKDGKKKKKKSKTKCVIM, +8 ), $\begin{array}{lll}\text { EKQTQ } & \text { (AAAGKKKKKKAQAQCVIM,+6), }\end{array}$ (AAAGKKQQKKAQAQCVIM, +4), EKAQ3Q (AAAGKQQQKKAQACVIM, +3) and EK6Q (SKDGQQQQQQSKTKCVIM, +2). The mRFP-Lact-C2 construct was a kind gift from Tony Yeung (University of Toronto) and was constructed by cloning the $\mathrm{C} 2$ domain of bovine lactadherin into mRFP-C1 vector [6]. This construct specifically recognizes phosphatidylserine containing membranes. The RaclWT-GFP [23], Rac1 tail-GFP (KKRKRKCLLL) [23]，Rac2WT-GFP，Rac1Q61L-GFP (Rac1CA) and Rac2Q61L (Rac2CA) [23] and HRAS-GFP [24] constructs were previously described. The Rac1-21-GFP and Rac2-1-2-GFP represent Rac1 and Rac2 proteins with interchanged polybasic sequence and preserved CAAX box [14] and were kind gifts from Dr. Naoaki Saito (Kobe University, Japan). The vector PH-PLC $\delta$-GFP $[25,26]$ encodes the PH domain of $\mathrm{PLC} \delta$ fused to EGFP (PH-PLC-GFP) and its construction has been described previously. The PH-AKT-GFP and PH-AKT-RFP encode the PH domains of protein kinase B fused to green or red fluorescent proteins and were also a kind gift from for Dr. Sergio Grinstein. The PX-P40-GFP [27] construct encodes the PX domain of p40-phox. All 
constructs were encoded in pcDNA3 or $\mathrm{C} 1$ plasmids and purified using QIAGEN Highspeed Maxi plasmid kit.

Neutrophil preparations and transfection. All procedures described were carried out in accordance with the Guide for the Humane Use and Care of Laboratory Animals and were approved by the University of Toronto Animal Care Committee. Neutrophil isolation was carried out as described previously [20]. Briefly, neutrophils were isolated from 6-10 weeks old C57BL/6J wild-type mice. Neutrophils were isolated from tibia and femur bone marrow using discontinuous Percoll (Sigma-Aldrich, Ontario, Canada) gradients of $82 \% / 65 \% / 55 \%$. More than $90 \%$ of cells isolated were mature neutrophils as assessed by Wright-Giemsa staining. Viability as determined by trypan blue exclusion was $>90 \%$. Bone marrow murine neutrophils were transfected using the protocol described previously $[9,21]$. Briefly, $3 \times 10^{6}$ primary neutrophils were resuspended in 100 $\mu 1$ nucleofactor solution $\mathrm{V}$ and supplemented with $5 \mu \mathrm{g}$ of the described DNA constructs. The cells were nucleoporated using the program Y-001 (Amaxa biosystems) and allowed to recover for 2 hours before the experiments. In experiments involving phagocytosis, $\sim 71 \%$ of transfected cells had at least 1 internalized bead after 30 minutes of phagocytosis. The transfection efficiency was analyzed by direct cell counting. Similar transfection efficiency was observed between the transfected probes (EK-GFP $-51 \% \pm$ 4.8\%, PH-AKT-GFP - 53\% \pm 6.9\%, Rac1Q61L-GFP - 44\% \pm 4.1\%, PH-PLC-GFP $47 \% \pm 4.9 \%$, Lact-C2-GFP $-52.2 \% \pm 3.9 \%$ ). The images were analyzed on a cell-by-cell basis in order to overcome expression levels variability or transfection efficiency. The differences in probe expression levels (based on MFI) were not significantly different. 
Fc-mediated phagocytosis. Transfected neutrophils were allowed to recover for 2 hours in DMEM supplemented with $10 \%$ FBS in non-tissue culture 6- well plates. $3.8 \mu \mathrm{m}$ Latex beads (Bangs Laboratories, USA) were coated with $20 \mathrm{mg} / \mathrm{ml}$ human IgG (Biodesign International) for 1 hour at $4{ }^{\circ} \mathrm{C}$. Neutrophils were spun down with the IgGcoated beads at $4^{\circ} \mathrm{C}$ for 30 seconds ( $9000 \mathrm{rpm}$ ), layered onto an un coated glass coverslip and mounted in an Attofluor live cell chamber (Invitrogen Canada). This was considered time 0. Cells were analyzed using a Leica DMIRE2 (Leica Microsystems Inc.) spinning disk confocal microscope equipped with a Hamamatsu Back-Thinned EM-CCD camera and the Volocity 4.2 platform. Cells were visualized at $100 \mathrm{X}$ with a $1.5 \mathrm{X}$ magnification lens (Spectral Applied Research) [21]. Pictures were taken every 10 seconds for 20 minutes. More than 200 cells were analyzed in seven independent experiments. The "cytoplasm" measurements comprised an average representative area of 1161 pixels of the cytoplasm. The pseudopod MFI contains the plasma membrane that stretches around the bead, but excludes the inner pseudopod membrane in contact with the bead. The rationale for this is the fact that as soon as the membrane contacts the bead, Rac1 and the +8 charge are dispersed. This is not due to probe quenching as the other probes are still visible at that stage.

Neutrophil chemotaxis. Transfected neutrophils were allowed to recover for 2 hours in DMEM supplemented with 10\% FBS in non-tissue culture 6- well plates. The cells were transferred to fibronectin-coated glass coverslip and mounted in an Attofluor live cell chamber (Invitrogen Canada). fMLP (N-formyl-methionine-leucine-phenylalanine) was 
delivered using an Eppendorf injectman micropipette at a concentration of $1 \mu \mathrm{M}$. Cells were analyzed using a spinning disk confocal microscope as described before. Pictures were taken every 6 seconds for 5 minutes. Alexa-647 cholera toxin B (Alexa-CTB; Molecular Probes, Invitrogen, Ontario, Canada) was used as a membrane marker. The ratio between the charge biosensors and the membrane marker was calculated using Volocity 4.3. A LUT filter was applied to the figures in order to visualize the results. All the cells were maintained at $37^{\circ} \mathrm{C}$ during the experiments.

Statistical analyses. Statistical analysis was performed using the SPSS 15.0 software (SPSS Inc.). Multiple comparisons were performed by ANOVA associated with the Bonferroni and Tukey's HSD tests for post hoc testing. All results represent at least three independent experiments. Statistical significance was defined as $P<0.05$. Data are expressed as mean \pm 1 standard error of the mean (SEM). All figures were created using Adobe Illustrator CS2. Photomicrographs were compensated for linear levels to remove background.

\section{Results}

Membrane phospholipid metabolism at the phagosome decreases net membrane charge 
To evaluate the distribution of membrane charges during phagocytosis, primary neutrophils were transfected with previously described fluorescently tagged charge biosensors that report the cytoplasmic surface charge of cellular membranes in real-time $[1,22]$. In neutrophils undergoing phagocytosis, the highly charged biosensors ( +6 and $+8)$ associated with the plasma membrane while the moderate to low-charge $(+2$ and +3$)$ probes associated with endomembranes (figure 1A). A clear decrease in the membrane charge negativity was observed from the plasma membrane towards the inner membranes. As shown in figure 1B, the highly charged +8 preferentially associate with the plasma membrane, while low-charge biosensors have a low plasma membrane affinity. Interestingly, the +4 biosensor highlights the phagosome membrane, while the low charge biosensors $(+2$ and +3$)$ display a more nonspecific binding to endomembranes, possibly golgi membrane and others. This is important to show that the phagosome membrane carries an intermediate membrane charge that is distinct from other endomembranes and the highly charged plasma membrane.

It has been demonstrated recently that membrane phospholipid composition determines the surface charge potential, which in turn regulates the localization of positively charged proteins $[6,7,22]$. In order to understand the observed differences in charge distribution during phagocytosis, we analyzed the dynamics of phospholipid localization during phagocytosis. Primary neutrophils were transfected with fluorescent probes for negatively charged lipids, including $\mathrm{PIP}_{2}, \mathrm{PIP}_{3}$ and PS (see Materials and Methods) and their localization was analyzed after 30 min of phagocytosis. The $\mathrm{PI}_{3} \mathrm{P}$ probe Px-P40-GFP was included in the analysis but was not found to be enriched at the phagosome membrane (data not shown). Figures $2 \mathrm{~A}$ and $\mathrm{B}$ show that $\mathrm{PIP}_{2}$ (PH-PLC- 
GFP) and $\mathrm{PIP}_{3}(\mathrm{PH}-\mathrm{AKT}-\mathrm{RFP})$ are enriched at the plasma membrane while PS (Lact-C2) is found at both the plasma membrane and phagosome membrane. The metabolism of the negatively charged plasma membrane lipids $\mathrm{PIP}_{2}$ and $\mathrm{PIP}_{3}$ that occurs during phagosome maturation resulted in reduced phagosome membrane negativity as highlighted by the release of the +8 charge probe (figure $2 \mathrm{~A}$ ). As shown in figure 2C, $\mathrm{PIP}_{2}$ (PH-PLC-GFP) is enriched at the pseudopods and rapidly dissociates from the newly formed phagosome membrane (figure 2C). In contrast, PS (Lact-C2-RFP) localization is unchanged as it is found both at the plasma membrane and phagosome membrane during all the stages of phagocytosis (figure 2D).

Distribution of net membrane charge during neutrophil phagocytosis: Tracking the localization of Rac1 and Rac2

The differences in the charge distribution throughout the cellular membranes led us to analyze the subcellular localization of active Rac1 and Rac2 as it has been demonstrated previously that Rac1 and Rac2 localize to separate membrane compartments during phagocytosis [29]. To accomplish this, constitutively active forms of Rac1 (Rac1CA) and Rac2 (Rac2CA) were transfected into neutrophils and analyzed in real-time during phagocytosis. Active Rac1 and Rac2 clearly differed in their localization during the progressing stages of phagocytosis (figure 3A and B). Rac1 was primarily found at the plasma membrane and pseudopods during phagosome formation, showing little association with the newly formed phagosome (Movie S1, Figure 3A), while Rac2 was weakly associated with the bead contact area but progressively accumulated to the base of the phagocytic cup and the fully developed phagosome ( $\sim 30$ minutes; figure $3 \mathrm{~B})$. 
It is clear from this data that Racl followed the +8 biosensor (figure $3 \mathrm{~A},+8$ panel) distribution pattern while Rac2 followed the $+3 /+4$ biosensor distribution (figure $3 \mathrm{~B},+3$ and +4 panels). The specific localization of Rac1 and Rac2 during different stages of phagocytosis is summarized in figure $3 \mathrm{C}$.

It has been reported that the C-terminal polybasic domain of small GTPases is responsible for the membrane domain targeting of these proteins $[13,14]$. To confirm the specific role polybasic sequence in Rac1 membrane targeting and to rule out other nonspecific binding sites, neutrophils were transfected with a construct carrying only the Rac polybasic sequence and anchoring box [30] (KKRKRKCLLL). The Rac1 tail probe showed clear plasma membrane recruitment with no association with the phagosome membrane (figure 3D), similar to the active Rac1 construct recruitment. As an additional control, we used Rac1 and Rac2 constructs carrying interchanged polybasic domain sequences but with their original prenylation domains (Rac1-2-1 and Rac2-1-2) [31]. This approach verified that the polybasic sequence influences the plasma membrane or phagosome membrane recruitment of Rac (figure 3E). The Rac1-2-1 construct matched the localization pattern of the Rac2 small GTPase and was found at both internal membranes and mature phagosome membranes, while Rac2-1-2 followed Rac1 and was preferentially associated with the plasma membrane (figure 3E). Taken together, the results confirm that the charge conveyed by the $\mathrm{PB}$ domain alone determines Rac isoform distribution.

A new insight into chemotaxis: The polarized accumulation of negative charges at the leading edge membrane 
Neutrophil chemotaxis is characterized by the polarization and migration up shallow chemical gradients. Our group and others have shown the importance of Rac small GTPases in this process, especially in the regulation of actin remodeling at the leading edge $[21,32]$. To assess the distribution of membrane charges during primary neutrophil migration, neutrophils were transfected with the +3 and +8 biosensors and analyzed in a micropipette delivered fMLP-mediated chemotaxis assay. As shown in figure $4 \mathrm{~A}$, the +8 charge biosensor immediately translocated to the leading edge membrane and membrane ruffling area closest to the chemoattractant source (5-fold increase compared to the membrane marker) and rapidly re-localized $(<12$ seconds) when the source was moved to another location (Movie S2 and S3). The +3 biosensor was mainly cytoplasmic and weakly translocates to the early forming leading edge area. Rac2CA did not accumulate at the plasma membrane and followed the +3 distribution pattern (data not shown). To further evaluate the charge dynamics of the leading edge membrane, the distribution of $\mathrm{PIP}_{2}, \mathrm{PIP}_{3}$ and $\mathrm{PS}$ were analyzed during neutrophil migration. In all experiments CTB was used as a membrane volume marker and its distribution was always uniform within the entire plasma membrane. In contrast to what was observed during phagocytosis, $\mathrm{PIP}_{3}$ increased at the leading edge membrane while there were no significant changes in $\mathrm{PIP}_{2}$ and PS (figure 4B and 4C). Importantly, even without ratioed analysis $\left(\mathrm{PIP}_{3} / \mathrm{CTB}\right)$, the increase of $\mathrm{PIP}_{3}$ at the leading edge is clearly evident (see supplemental movie S4). This accumulation of the negatively charged $\mathrm{PIP}_{3}$ lipid in the leading edge membrane explains the translocation of the +8 biosensor to the leading edge membrane. In order to confirm the role of $\mathrm{PIP}_{3}$ in the polarization of the charges towards the leading edge, neutrophils were transfected with the +8 biosensor and 
treated with $100 \mathrm{nM}$ Wortmannin. Figure 4D shows that wortmannin blocked the accumulation of +8 at the leading edge. We describe here for the first time that neutrophils have a polarized plasma membrane charge distribution during chemotaxis and the accumulation of negatively charged $\mathrm{PIP}_{3}$ at the leading edge membrane results in the recruitment of highly charged proteins including Rac1 (figure 4E).

\section{Discussion}


The goal of this study was to determine the distribution of membrane charge in primary neutrophils during chemotaxis and phagocytosis and determine whether changes in membrane charge contributes to the differential localization of Rac small GTPases to specific cellular membranes. We describe here that during phagocytosis the partial depletion of specific membrane phospholipids $\left(\mathrm{PIP}_{2}, \mathrm{PIP}_{3}\right)$ in the phagosome membrane decreases the net membrane charge. Importantly, the persistence of PS in the phagosome membrane generates a moderately charged membrane environment that results in the preferential localization of Rac2. During chemotaxis, neutrophils show increased membrane negativity at the leading edge due to the accumulation of $\mathrm{PIP}_{3}$. Through these two neutrophil processes we demonstrate that electrostatic forces between negative membrane lipids and the positive amino acid residues in the $\mathrm{C}$-terminal of the proteins influence the localization patterns of Rac1 and Rac2 to distinct membrane compartments which adds further insight into the mechanisms regulating small GTPase localization.

\section{Charge-dependent membrane targeting in Rac small GTPases}

Efficient membrane localization and activation of small GTPases is a complex process that involves RhoGDIs, GEFs, GAPs and other binding partners that may influence the localization of small GTPases. The competition for proteins to bind to specific membranes depends on several factors, including the presence of specific lipid binding motifs, protein-protein interactions and lipid charge interactions.

Our principal question was whether the unique polybasic domain charge of Rac isoforms drives the specific phospholipid-dependent membrane charge distribution in primary neutrophils during chemotaxis and phagocytosis. The strikingly different roles of 
the Rac isoforms during these processes in neutrophils have been reported previously by our group and others $[20,21,33]$. It is also notable that the polybasic sequences of Rac help determine their function [14, 23, 34]. Using an in vitro model Ugolev et al [35] assessed Rac1 and Rac2 dissociation from RhoGDI in liposomes carrying anionic lipids. They showed that the less charged Rac2 was more resistant to dissociation from the RhoGDI complexes than Rac1 possibly due to the charge differences in the polybasic domain. Further support for this mechanism comes from Tao et al [36] who used NIH3T3 fibroblasts to show that Rac2 constructs missing the polybasic sequence (TRQQKRP) fail to localize properly. Furthermore Yamauchi et al [31] used Rac2 constructs to show that the polybasic sequence is the key determinant for rescuing oxidase activity and migration in Rac2 null cells. The aforementioned studies did not explore how neutrophils regulate the phospholipid-dependent charge distribution during phagocytosis or chemotaxis. More specifically, how the membrane phospholipid composition may impact the electrostatic affinities that differentially localizes Rac isoforms.

Using primary neutrophils, we provide evidence that Rac proteins localize to specific membranes domain based on the alterations in membrane lipid composition, which affects electrostatic interactions between the Rac polybasic domain and the cellular membranes. The depletion of specific phospholipids at the phagosome generates an intermediate negative charge that recruits intermediately charged proteins, e.g. Rac2. In addition to that, the polarized accumulation of phospholipids at a specific membrane region (leading edge) selectively recruits highly charged proteins, e.g. Rac1. Phospholipid metabolism during processes such as phagocytosis is responsible for recruiting new effector proteins and sending away those that have already played their 
role. Our results are also in line with others that investigated the charge-dependent localization of Rac [14]. Importantly, in our primary neutrophil model, we show that Rac2 but not Rac1 is recruited to the phagosome membrane and this recruitment is associated with the accumulation of PS and an intermediately charged environment. Several other publications support the observations described here. It has been previously demonstrated that Rac1 constructs carrying non-charged aminoacid substitutions at the polybasic domain fail to localize to the plasma membrane [18]. In addition, the scrambling of the basic aminoacids at the polybasic domain of Rac did not prevent membrane localization confirming that it is the net charge and not the sequence of the charged amino acids that is critical [18]. Heo et al [5] have recently shown that the presence of both $\mathrm{PIP}_{2}$ and $\mathrm{PIP}_{3}$ are necessary for the recruitment of small GTPases carrying either prenyl, farnesyl or geranyl anchoring sites at the plasma membrane of NIH3T3 and Hela cells. Although the authors suggest that the presence of $\mathrm{PIP}_{2}$ and $\mathrm{PIP}_{3}$ is the key element regulating the recruitment of these proteins, several other studies have shown that more abundant negative lipids like PS are equally important in the targeting of positively charged proteins [6]. As a case in point, previous work by the Grinstein group has demonstrated the important role of phosphatidylserine in generating the membrane negativity necessary for the recruitment of positively charged proteins $[6,18]$. In the cellular milieu, when the membrane charge decreases, moderately charged proteins effectively compete with highly charged proteins for binding sites which in turn explains the differential localization observed based on protein charge [6].

The proposed charge-dependent Rac Localization Model 
Our findings for Fc-mediated phagocytosis are summarized in figure 5A-D. Upon receptor activation, $\mathrm{PIP}_{2}$ hydrolysis and PS externalization results in a decreased membrane charge at the bead contact area. Meanwhile, actin-rich pseudopods and the adjacent plasma membrane accumulate $\mathrm{PS}, \mathrm{PIP}_{2}$ and $\mathrm{PIP}_{3}$ as highlighted by the +8 charge (figure 5A-B). Figure 5C and 5D show that upon phagosome sealing and internalization, the metabolism of phospholipids results in net charge loss and the association of Rac2 with the phagosome while Rac1, PS, $\mathrm{PIP}_{2}$ and the +8 charge probe immediately translocated back to the plasma membrane. Up to 30 minutes after internalization, Rac2 was still found to be associated with the phagosome. This charge shift mechanism may be important in the regulation of phagocytosis and bacterial killing, including the generation of ROS at the phagosome, a process that is regulated by Rac2 in neutrophils [37]. Importantly, we show that $\mathrm{PIP}_{3}$ is depleted from the late stage phagosome of neutrophils ( $>15$ min minutes). Although, others have shown that $\mathrm{PIP}_{3}$ is required for Fc-mediated phagocytosis particularly during phagosomes formation, marked by an early transient accumulation of $\mathrm{PIP}_{3}(<1 \mathrm{~min})$ before cup sealing [38] our report is consistent with others that show the depletion of $\mathrm{PIP}_{3}$ in the late stage phagosome (after phagosome sealing) [39].

\section{Charge polarization during chemotaxis}

We also demonstrate here that neutrophil polarization and chemotaxis is accompanied by an increase in leading edge membrane negativity and that this mechanism selectively recruits highly charged molecules to the front of the cell (figure 5E). This mechanism depends on the accumulation of $\mathrm{PIP}_{3}$ at the leading edge and further 
highlights the role of PI3K activity during early stages of neutrophil chemotaxis. Earlier studies showed that the activation of Rac and an associated positive feedback loop with PI3 kinase amplifies and stabilizes shallow gradients of extracellular signals that are crucial for establishing polarity in neutrophils when they are activated by chemoattractants $[40,41]$. Moreover, the inhibition of PI3K decreased the cells ability to interpret chemoattractant gradients [40]. Conversely, recent studies have shown that neutrophils lacking PI3K were still able to migrate towards chemoattractants [42]. The same group reported that PI3K-independent pathways, including the TorC2 and the PLA2 pathways, were likely to mediate chemotaxis downstream of $\mathrm{G}$ coupled receptor activation [42-44]. Despite this controversy, it is largely accepted that Rac small GTPases and PI3K products play an important role in cell migration and the idea of a chargedependent mechanism involving PIP $_{3}$ may be an additional mechanism to support the currently accepted Rac1-GEF-PI3K activation cycle [45]. Our observations are also in agreement with a recent publication by Costa et. al. that elegantly showed that constitutively and delocalized $\mathrm{PIP}_{3}$ production impairs leading edge formation and directional migration through a Rac1 dependent mechanism [44].

\section{Limitations of the study}

Rac is normally found in the cytoplasm associated with RhoGDI [23] in an inactive state with a small fraction associated with membranes. Upon release from RhoGDIs, small GTPases translocate to membrane compartments for activation. The charge mechanism described here would hypothetically impact on the ability of free Rac1 to bind and stay at negatively charged membranes rather than lower charge membranes 
(e.g. phagosome membrane). It is not the aim of this work to account for all the regulatory mechanisms that impact protein localization. However it is clear that electrostatic affinity due to phospholipids plays an important role in active small GTPase localization. The rationale for using constitutively active Rac constructs as opposed to non-mutated Rac constructs was based on several points. First, constitutively active Rac (CA-Rac) was used because changes in its' membrane association can't be attributed to more or less GEF or GAP activity. Also, CA-Rac is mainly membrane associated and its localization is not affected by RhoGDI binding, which enhances the ability to visualize its localization in real time. We recognize that the localization of constitutively active constructs does not mimic physiological signaling pathways but it did allow us to address our primary objective by controlling for other contributing factors that would have complicated the data analysis.

\section{Summary}

The present work provides evidence that neutrophils may control protein localization and the activation of signaling pathways through the regulation of phospholipid metabolism and the resulting alteration in membrane net charge. This finding is of great interest considering recent reports describing bacterial proteins that can modulate their own survival by interfering with phagosomal membrane lipid metabolism [46]. Additionally, it expands the current accepted charge-dependent model by showing that proteins carrying intermediate positive charges can be selectively recruited to 
specific internal membranes. This mechanism may help us understand the mechanisms underlying effective neutrophil activation during innate immunity.

\section{Acknowledgements}

We gratefully thank Dr. Sergio Grinstein and Tony Yeung (Hospital for Sick Children) for sharing the reagents and helpful discussions. We also thank Dr. Richard Ellen 
(University of Toronto) and Dr. Karl-Eric Magnusson (Linköping University) for helpful comments.

\section{Authorship}

Contribution: MAOM designed and performed the experiments, analyzed the data and wrote the manuscript. MG designed the research and edited the manuscript.

Conflict-of-interest disclosure: The authors declare no competing financial interests.

\section{References}


1. Yeung, T., Grinstein, S. (2007) Lipid signaling and the modulation of surface charge during phagocytosis. Immunol Rev 219, 17-36.

2. McLaughlin, S. (1989) The electrostatic properties of membranes. Annu Rev Biophys Biophys Chem 18, 113-36.

3. Verkleij, A.J., Post, J.A. (2000) Membrane phospholipid asymmetry and signal transduction. J Membr Biol 178, 1-10.

4. Quinn, P.J. (2002) Plasma membrane phospholipid asymmetry. Subcell Biochem 36, 39-60.

5. Heo, W.D., Inoue, T., Park, W.S., Kim, M.L., Park, B.O., Wandless, T.J., Meyer, T. (2006) $\mathrm{PI}(3,4,5) \mathrm{P} 3$ and $\mathrm{PI}(4,5) \mathrm{P} 2$ lipids target proteins with polybasic clusters to the plasma membrane. Science 314, 1458-61.

6. Yeung, T., Gilbert, G.E., Shi, J., Silvius, J., Kapus, A., Grinstein, S. (2008) Membrane phosphatidylserine regulates surface charge and protein localization. Science 319, 210-3.

7. McLaughlin, S., Aderem, A. (1995) The myristoyl-electrostatic switch: a modulator of reversible protein-membrane interactions. Trends Biochem Sci 20, 272-6.

8. Kobayashi, S.D., Voyich, J.M., Burlak, C., DeLeo, F.R. (2005) Neutrophils in the innate immune response. Arch Immunol Ther Exp (Warsz) 53, 505-17.

9. Magalhaes, M.A., Zhu, F., Sarantis, H., Gray-Owen, S.D., Ellen, R.P., Glogauer, M. (2007) Expression and translocation of fluorescent-tagged p21-activated kinase-binding domain and $\mathrm{PH}$ domain of protein kinase $\mathrm{B}$ during murine neutrophil chemotaxis. J Leukoc Biol 82, 559-66.

10. Kreck, M.L., Uhlinger, D.J., Tyagi, S.R., Inge, K.L., Lambeth, J.D. (1994) Participation of the small molecular weight GTP-binding protein Rac1 in cell-free activation and assembly of the respiratory burst oxidase. Inhibition by a carboxylterminal Rac peptide. J Biol Chem 269, 4161-8.

11. Joseph, G., Gorzalczany, Y., Koshkin, V., Pick, E. (1994) Inhibition of NADPH oxidase activation by synthetic peptides mapping within the carboxyl-terminal domain of small GTP-binding proteins. Lack of amino acid sequence specificity and importance of polybasic motif. J Biol Chem 269, 29024-31.

12. Missy, K., Van Poucke, V., Raynal, P., Viala, C., Mauco, G., Plantavid, M., Chap, H., Payrastre, B. (1998) Lipid products of phosphoinositide 3-kinase interact with Rac1 GTPase and stimulate GDP dissociation. J Biol Chem 273, 30279-86.

13. Sigal, N., Gorzalczany, Y., Pick, E. (2003) Two pathways of activation of the superoxide-generating NADPH oxidase of phagocytes in vitro--distinctive effects of inhibitors. Inflammation 27, 147-59.

14. Ueyama, T., Eto, M., Kami, K., Tatsuno, T., Kobayashi, T., Shirai, Y., Lennartz, M.R., Takeya, R., Sumimoto, H., Saito, N. (2005) Isoform-specific membrane targeting mechanism of Rac during Fc gamma R-mediated phagocytosis: positive charge-dependent and independent targeting mechanism of Rac to the phagosome. J Immunol 175, 2381-90.

15. Williams, C.L. (2003) The polybasic region of Ras and Rho family small GTPases: a regulator of protein interactions and membrane association and a site of nuclear localization signal sequences. Cell Signal 15, 1071-80. 
16. Finkielstein, C.V., Overduin, M., Capelluto, D.G. (2006) Cell migration and signaling specificity is determined by the phosphatidylserine recognition motif of Rac1. J Biol Chem 281, 27317-26.

17. Lanning, C.C., Daddona, J.L., Ruiz-Velasco, R., Shafer, S.H., Williams, C.L. (2004) The Rac1 C-terminal polybasic region regulates the nuclear localization and protein degradation of Rac1. J Biol Chem 279, 44197-210.

18. Yeung, T., Terebiznik, M., Yu, L., Silvius, J., Abidi, W.M., Philips, M., Levine, T., Kapus, A., Grinstein, S. (2006) Receptor activation alters inner surface potential during phagocytosis. Science 313, 347-51.

19. Hancock, J.F., Paterson, H., Marshall, C.J. (1990) A polybasic domain or palmitoylation is required in addition to the CAAX motif to localize p21 ras to the plasma membrane. Cell 63, 133-9.

20. Sun, C.X., Downey, G.P., Zhu, F., Koh, A.L., Thang, H., Glogauer, M. (2004) Racl is the small GTPase responsible for regulating the neutrophil chemotaxis compass. Blood 104, 3758-65.

21. Sun, C.X., Magalhaes, M.A., Glogauer, M. (2007) Rac1 and Rac2 differentially regulate actin free barbed end formation downstream of the fMLP receptor. $J$ Cell Biol 179, 239-45.

22. Roy, M.O., Leventis, R., Silvius, J.R. (2000) Mutational and biochemical analysis of plasma membrane targeting mediated by the farnesylated, polybasic carboxy terminus of K-ras4B. Biochemistry 39, 8298-307.

23. Michaelson, D., Silletti, J., Murphy, G., D'Eustachio, P., Rush, M., Philips, M.R. (2001) Differential localization of Rho GTPases in live cells: regulation by hypervariable regions and RhoGDI binding. J Cell Biol 152, 111-26.

24. Choy, E., Chiu, V.K., Silletti, J., Feoktistov, M., Morimoto, T., Michaelson, D., Ivanov, I.E., Philips, M.R. (1999) Endomembrane trafficking of ras: the CAAX motif targets proteins to the ER and Golgi. Cell 98, 69-80.

25. Stauffer, T.P., Ahn, S., Meyer, T. (1998) Receptor-induced transient reduction in plasma membrane PtdIns(4,5)P2 concentration monitored in living cells. Curr Biol 8, 343-6.

26. Varnai, P., Balla, T. (1998) Visualization of phosphoinositides that bind pleckstrin homology domains: calcium- and agonist-induced dynamic changes and relationship to myo-[3H]inositol-labeled phosphoinositide pools. J Cell Biol 143, 501-10.

27. Kanai, F., Liu, H., Field, S.J., Akbary, H., Matsuo, T., Brown, G.E., Cantley, L.C., Yaffe, M.B. (2001) The PX domains of p47phox and p40phox bind to lipid products of PI(3)K. Nat Cell Biol 3, 675-8.

28. Kenworthy, A.K., Nichols, B.J., Remmert, C.L., Hendrix, G.M., Kumar, M., Zimmerberg, J., Lippincott-Schwartz, J. (2004) Dynamics of putative raftassociated proteins at the cell surface. $J$ Cell Biol 165, 735-46.

29. Hoppe, A.D., Swanson, J.A. (2004) Cdc42, Rac1, and Rac2 display distinct patterns of activation during phagocytosis. Mol Biol Cell 15, 3509-19.

30. Michaelson, D., Ahearn, I., Bergo, M., Young, S., Philips, M. (2002) Membrane trafficking of heterotrimeric $\mathrm{G}$ proteins via the endoplasmic reticulum and Golgi. Mol Biol Cell 13, 3294-302. 
31. Yamauchi, A., Marchal, C.C., Molitoris, J., Pech, N., Knaus, U., Towe, J., Atkinson, S.J., Dinauer, M.C. (2005) Rac GTPase isoform-specific regulation of NADPH oxidase and chemotaxis in murine neutrophils in vivo. Role of the Cterminal polybasic domain. $J$ Biol Chem 280, 953-64.

32. Glogauer, M., Hartwig, J., Stossel, T. (2000) Two pathways through Cdc42 couple the $\mathrm{N}$-formyl receptor to actin nucleation in permeabilized human neutrophils. J Cell Biol 150, 785-96.

33. Glogauer, M., Marchal, C.C., Zhu, F., Worku, A., Clausen, B.E., Foerster, I., Marks, P., Downey, G.P., Dinauer, M., Kwiatkowski, D.J. (2003) Rac1 deletion in mouse neutrophils has selective effects on neutrophil functions. J Immunol 170, 5652-7.

34. Filippi, M.D., Harris, C.E., Meller, J., Gu, Y., Zheng, Y., Williams, D.A. (2004) Localization of Rac2 via the $\mathrm{C}$ terminus and aspartic acid 150 specifies superoxide generation, actin polarity and chemotaxis in neutrophils. Nat Immunol 5, 744-51.

35. Ugolev, Y., Molshanski-Mor, S., Weinbaum, C., Pick, E. (2006) Liposomes comprising anionic but not neutral phospholipids cause dissociation of $\operatorname{Rac}(1$ or 2) $x$ RhoGDI complexes and support amphiphile-independent NADPH oxidase activation by such complexes. J Biol Chem 281, 19204-19.

36. Tao, W., Filippi, M.D., Bailey, J.R., Atkinson, S.J., Connors, B., Evan, A., Williams, D.A. (2002) The TRQQKRP motif located near the C-terminus of Rac2 is essential for Rac2 biologic functions and intracellular localization. Blood 100, $1679-88$.

37. Roberts, A.W., Kim, C., Zhen, L., Lowe, J.B., Kapur, R., Petryniak, B., Spaetti, A., Pollock, J.D., Borneo, J.B., Bradford, G.B., Atkinson, S.J., Dinauer, M.C., Williams, D.A. (1999) Deficiency of the hematopoietic cell-specific Rho family GTPase Rac2 is characterized by abnormalities in neutrophil function and host defense. Immunity 10, 183-96.

38. Vieira, O.V., Botelho, R.J., Rameh, L., Brachmann, S.M., Matsuo, T., Davidson, H.W., Schreiber, A., Backer, J.M., Cantley, L.C., Grinstein, S. (2001) Distinct roles of class I and class III phosphatidylinositol 3-kinases in phagosome formation and maturation. J Cell Biol 155, 19-25.

39. Marshall, J.G., Booth, J.W., Stambolic, V., Mak, T., Balla, T., Schreiber, A.D., Meyer, T., Grinstein, S. (2001) Restricted accumulation of phosphatidylinositol 3kinase products in a plasmalemmal subdomain during Fc gamma receptormediated phagocytosis. J Cell Biol 153, 1369-80.

40. Wang, F., Herzmark, P., Weiner, O.D., Srinivasan, S., Servant, G., Bourne, H.R. (2002) Lipid products of PI(3)Ks maintain persistent cell polarity and directed motility in neutrophils. Nat Cell Biol 4, 513-8.

41. Servant, G., Weiner, O.D., Herzmark, P., Balla, T., Sedat, J.W., Bourne, H.R. (2000) Polarization of chemoattractant receptor signaling during neutrophil chemotaxis. Science 287, 1037-40.

42. Kamimura, Y., Xiong, Y., Iglesias, P.A., Hoeller, O., Bolourani, P., Devreotes, P.N. (2008) PIP3-independent activation of TorC2 and PKB at the cell's leading edge mediates chemotaxis. Curr Biol 18, 1034-43. 
43. Janetopoulos, C., Firtel, R.A. (2008) Directional sensing during chemotaxis. FEBS Lett 582, 2075-85.

44. Costa, C., Barberis, L., Ambrogio, C., Manazza, A.D., Patrucco, E., Azzolino, O., Neilsen, P.O., Ciraolo, E., Altruda, F., Prestwich, G.D., Chiarle, R., Wymann, M., Ridley, A., Hirsch, E. (2007) Negative feedback regulation of Rac in leukocytes from mice expressing a constitutively active phosphatidylinositol 3-kinase gamma. Proc Natl Acad Sci U S A 104, 14354-9.

45. Kolsch, V., Charest, P.G., Firtel, R.A. (2008) The regulation of cell motility and chemotaxis by phospholipid signaling. J Cell Sci 121, 551-9.

46. Steinberg, B.E., Grinstein, S. (2008) Pathogen destruction versus intracellular survival: the role of lipids as phagosomal fate determinants. J Clin Invest 118, 2002-11.

Figure legends 
Figure 1. Analysis of membrane charge distribution during neutrophil phagocytosis.

(A) The negative charge distribution of neutrophil membranes increases from the perinuclear area towards the plasma membrane. Murine neutrophils were transfected with the described charge biosensor fluorescent probes and subjected to IgG-coated latex bead phagocytosis. The cells were allowed to phagocytose for $30 \mathrm{~min}$ and images were taken using a spinning disk confocal microscope. Images shown are representative of a total of 70 cells analyzed in four independent experiments. (B) The more positively charged biosensors preferentially associate with the more negatively charged plasma membrane. The mean fluorescence intensity (MFI) of the plasma membrane and the average cytosolic area was calculated using the Volocity 4.2 platform. The bars represent the average membrane/cytoplasm MFI ratio. The GFP and HRAS vectors were used as controls. Results are based on the analysis of at least 10 cells per group in multiple experiments.

Figure 2. Phospholipid metabolism at the phagosome membrane decreases the surface charge. (A) Phagosome membrane is partially depleted of membrane phospholipids and is less negatively charged. Neutrophils were transfected with PHAKT-RFP (PIP3), PH-PLC-GFP (PIP2), LACT-C2-GFP (PS) and EK-GFP (+8 biosensor) and the localization of the phospholipids was analyzed after 30 minutes of neutrophil phagocytosis. The plasma membrane and phagosome membrane mean fluorescence intensity (MFI) of each cell was measured and normalized to the cytoplasmic MFI. (PH-PLC, $\mathrm{n}=8, \mathrm{P}<0.011$; PH-AKT, $\mathrm{n}=10, \mathrm{P}<0.001$; LACT-C2, $\mathrm{n}=50$, $\mathrm{P}<0.020$ ). (B) Representative images of the distribution of the fluorescent probes 
measured on panel A. (C) PIP2 dissociated from the phagosome membrane at the early stages of phagocytosis. Neutrophils transfected with the described probes were allowed to phagocytose IgG-coated latex beads (see materials and methods). Images shown are snapshots of a time-lapse microscopy movie and are representative of a total of 15 cells. The representative areas 1(Cup base), 2 (Pseudopods), 3 (early phagosome membrane) and 4 (Near plasma membrane) highlighted in the images were analyzed for the relative MFI of the probes. (D) Phosphatidylserine persists at the phagosome membrane after 15 min of phagocytosis. Neutrophils transfected with the Lact-C2 RFP probe were subjected to the phagocytosis protocol described before. Images shown are snapshots of a timelapse microscopy movie and are representative of a total of 15 cells in 5 independent experiments. Scale bar $=12 \mu \mathrm{M}$.

\section{Figure 3. Membrane charges dictate the localization of Rac1 and Rac2 during} phagocytosis. (A-B) Rac1CA follows the distribution pattern of the +8 probe while Rac2CA localization is similar to the $+3 /+4$ probe during phagocytosis. Neutrophils were transfected with $+3,+4,+8$, Rac1CA and Rac2CA constructs and their localization was analyzed during 3 different stages of phagocytosis (early contact, cup phase and detached phagosome). Transfected neutrophils were mounted on a live cell chamber and submitted to an IgG-coated latex bead phagocytosis protocol. Images are representative of 39 cells analyzed in 3 separate experiments. Asterisks mark the bead location. Scale bar $=12 \mu \mathrm{m}$.

(C) Rac1 and Rac2 show differential accumulation during the progression of phagocytosis. For each probe, the mean fluorescence intensity of the described areas was 
measured and compared to the MFI of the plasma membrane. Results were plotted on the same graph to facilitate the comparison. The "pseudopod membrane" comprises the plasma membrane stretching around the bead, excluding the membrane in direct contact with the bead. Results are based on the analysis of 31 cells in 4 independent experiments. $\mathrm{P}<0.004$. (D) Rac1 localization is determined by the PB domain alone. Neutrophils were transfected with a construct carrying the Rac1 polybasic sequence and the CAAX membrane-binding motif. The cells were subjected to the described phagocytosis protocol and protein localization was analyzed in real time. (E) Rac1 and Rac2 polybasic sequences dictate their charge-dependent membrane localization. Neutrophils were transfected with Rac1WT, Rac2WT and Rac constructs with interchanged polybasic sequences denoted as Rac1-2-1 and Rac2-1-2. The cells were subjected to IgG-coated latex bead phagocytosis and analyzed in real time using a spinning disk confocal microscope. Images were acquired 30 minutes after phagocytosis and the MFI was calculated using Volocity 4.2. Results are based on the analysis of 28 cells in 3 independent experiments. ( $n=3, P<0.001$ - between Rac121 and Rac212)

Figure 4. Generation of a charge gradient during neutrophil chemotaxis. (A) Probes carrying a strong cationic net charge are recruited to the leading edge membrane during neutrophil chemotaxis. Neutrophils were transfected with the EK-GFP $(+8)$ probe and EKAQ3Q-GFP (+3) and subjected to a micropipette delivered fMLP-mediated chemotaxis assay. The asterisks represent the source of chemoattractant ( $1 \mu \mathrm{M}$ fMLP). Only cells that had a clear leading edge formed towards the source of chemoattractant 
and actively migrated were considered for this experiment. Images were taken every 6 seconds for 5 minutes using a spinning disk confocal microscope. Data based on the analysis of 30 cells in three independent experiments. The Alexa-CTB 647 dye was used as a membrane marker and to normalize the GFP channel. For each timepoint a ratio between the charge biosensors fluorescence intensity and the membrane marker fluorescence intensity was generated (right panels) and shown as a LUT scale. Scale bar $=10 \mu \mathrm{m}$. (B) $\mathrm{PIP}_{3}$ accumulates at the leading edge of migrating neutrophils. Neutrophils were transfected with PH-AKT-RFP, PH-PLC-GFP and LACT-C2 and subjected to the chemotaxis assay described above. The MFI of the lipid probes was measured at the leading edge membrane and trailing edge membrane and compared at each cell. Results were normalized using the average cytoplasmic fluorescence intensity and based on the analysis of 12 cells per group in three independent observations. ( $\mathrm{P}<0.01-\mathrm{PH}-\mathrm{AKT}$-RFP groups). (C) Distribution of $\mathrm{PIP}_{2}, \mathrm{PIP}_{3}$ and PS after fMLP stimulation. Neutrophils transfected with the ratio between the PH-AKT-RFP, PH-PLC-GFP and LACT-C2 lipid probes and subjected to the chemotaxis assay described on panel A. Te asterisks represent the source of chemoattractant. The bottom images represent a ratio between the lipid biosensor and the membrane marker and was generated using Volocity 4.2 and shown using a LUT scale. (D) PI3K inhibition decreases the accumulation of the +8 probe at the leading edge. Neutrophils were transfected with the EK $(+8)$ construct and subjected to micropipette-delivered fMLP chemotaxis. Cells polarizing and actively moving towards the pipette were analyzed. Where indicated, 100nM Wortmannin was added. Results are shown as the membrane MFI compared to the cytoplasm and are based on the analysis of 60 cells in three different experiments $(\mathrm{P}<0.032$, ANOVA). (E) Active Rac1 is recruited 
to the leading edge membrane. Neutrophils expressing constitutively active Rac1 were subjected to the chemotaxis experiment described previously. A ratio between Rac1 and the membrane marker was generated to illustrate the accumulation of Rac1 at the leading edge. Asterisk represents the source of chemoattractant. Scale bar $=10 \mu \mathrm{m}$. Results represent more than 30 cells in three independent experiments.

Figure 5. Membrane charge regulation in neutrophils. (A-D) Representation of the membrane charge distribution during different stages of neutrophil phagocytosis. The receptor activation following bead contact induces lipid metabolism and the decrease in the negativity of the membrane in the maturing phagosome. The base of the phagocytic cup and the phagosome membrane are increasingly depleted of phospholipids and are associated with intermediately charged biosensors. During phagocytosis, Rac1 is associated with highly charged actin-rich pseudopods and plasma membrane while Rac2 is mostly associated with the lowly charged phagosome membrane. (E) Representation of the membrane charge regulation during neutrophil chemotaxis. Neutrophil chemotaxis is accompanied by the increase in the negativity of the leading edge membrane and the recruitment of highly charged proteins.

Movie S1. Active Rac1 accumulates at the plasma membrane and pseudopods during phagocytosis.

Movie S2 and S3. The +8 biosensor (Green) accumulates at the leading edge membrane of migrating neutrophils (Ratio)

Movie S4. PH-AKT-GFP accumulates at the leading edge of migrating neutrophils. 
Figure 1

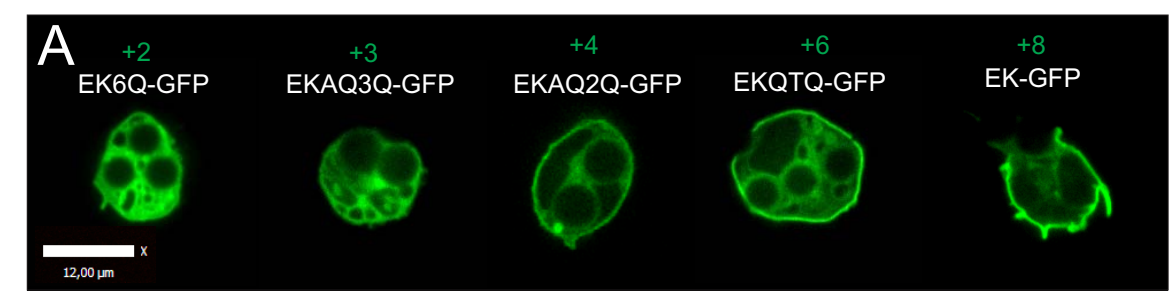

$\mathrm{B} \stackrel{\circ}{=}$ Distribution of fluorescent probes (Plasma membrane)

B

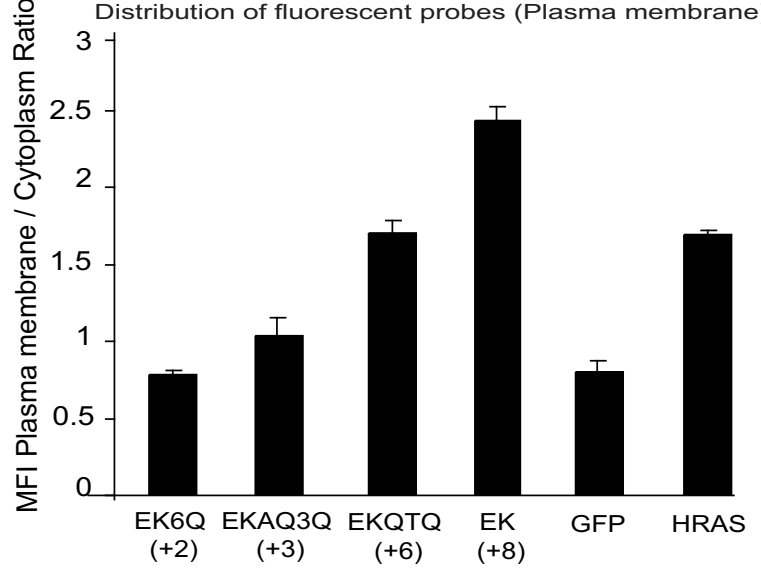



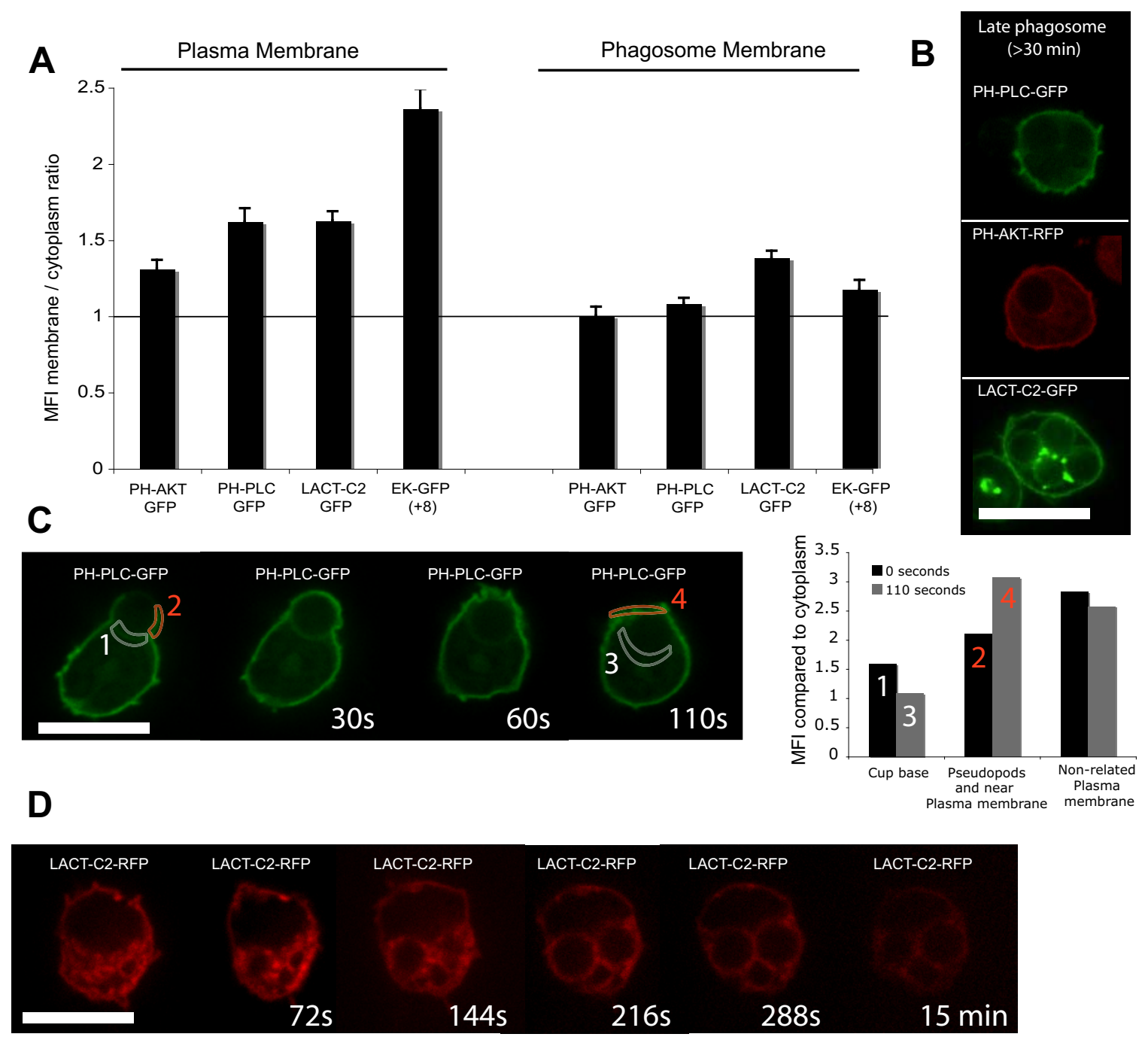
Figure 3

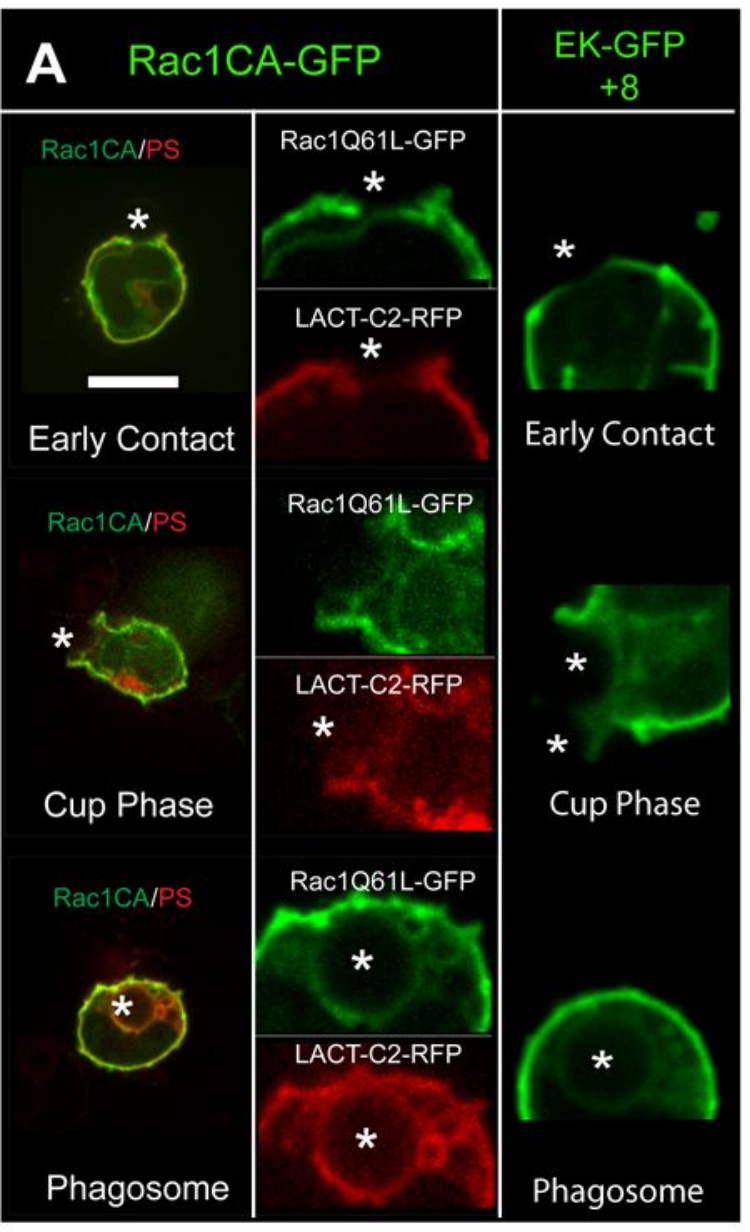

Distribution of Fluorescent probes

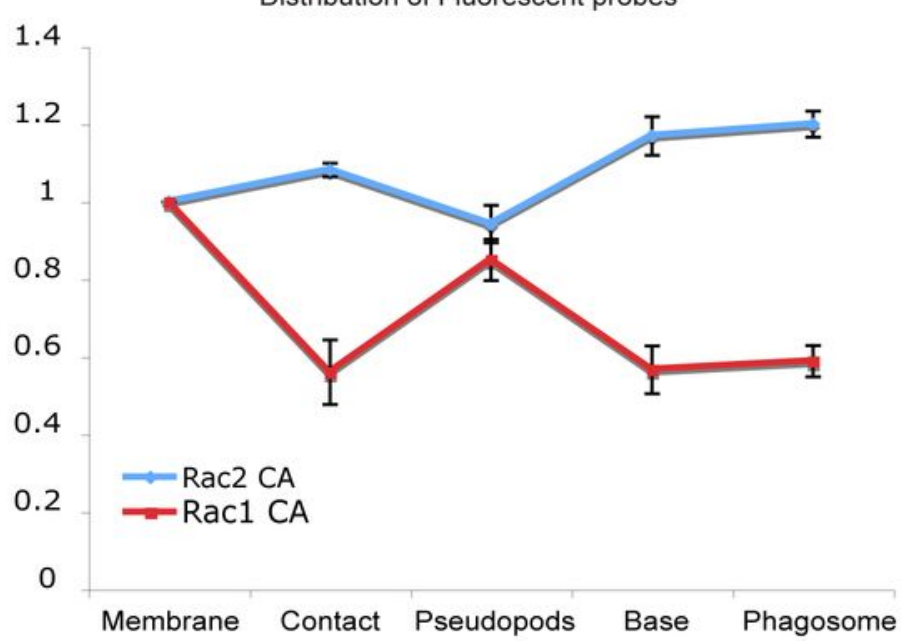

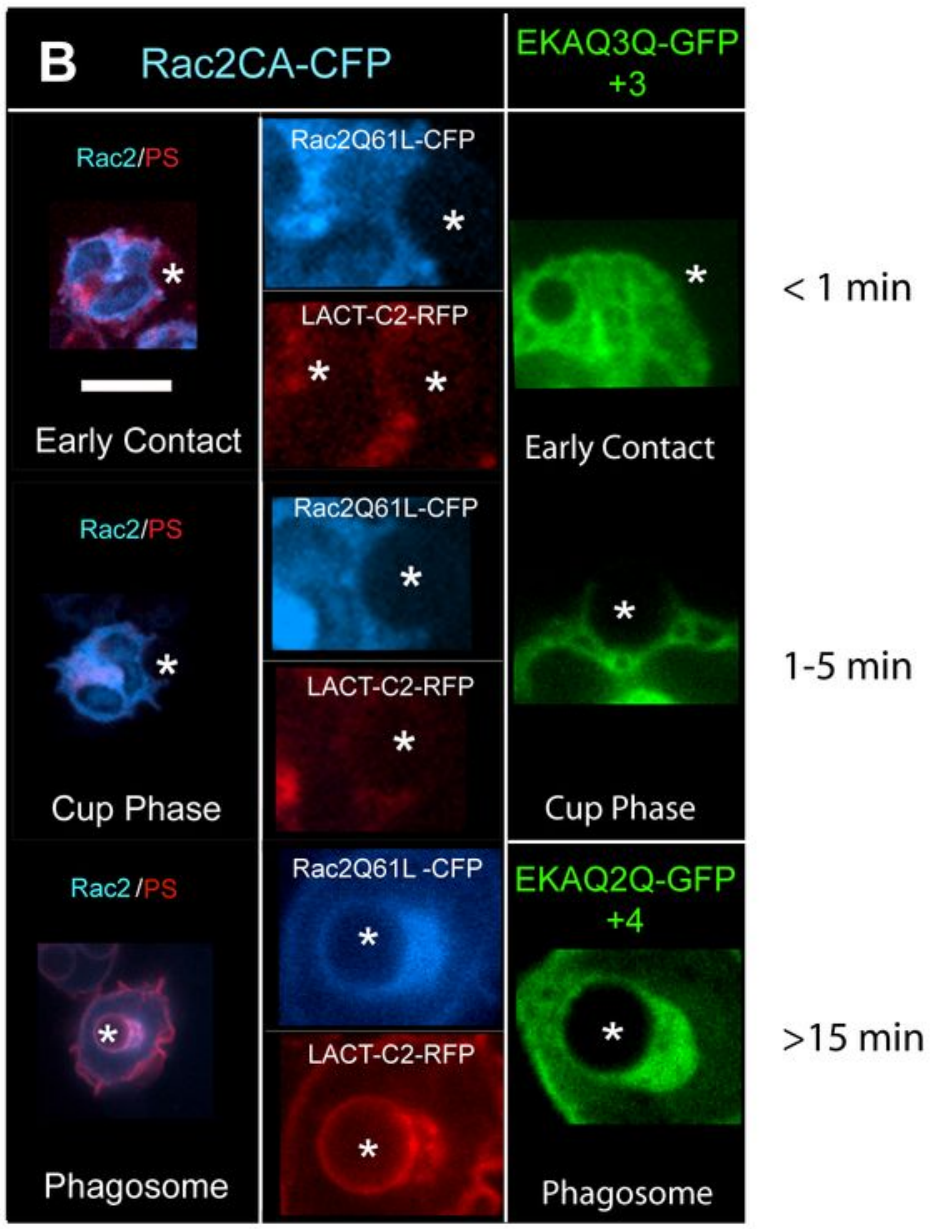

E

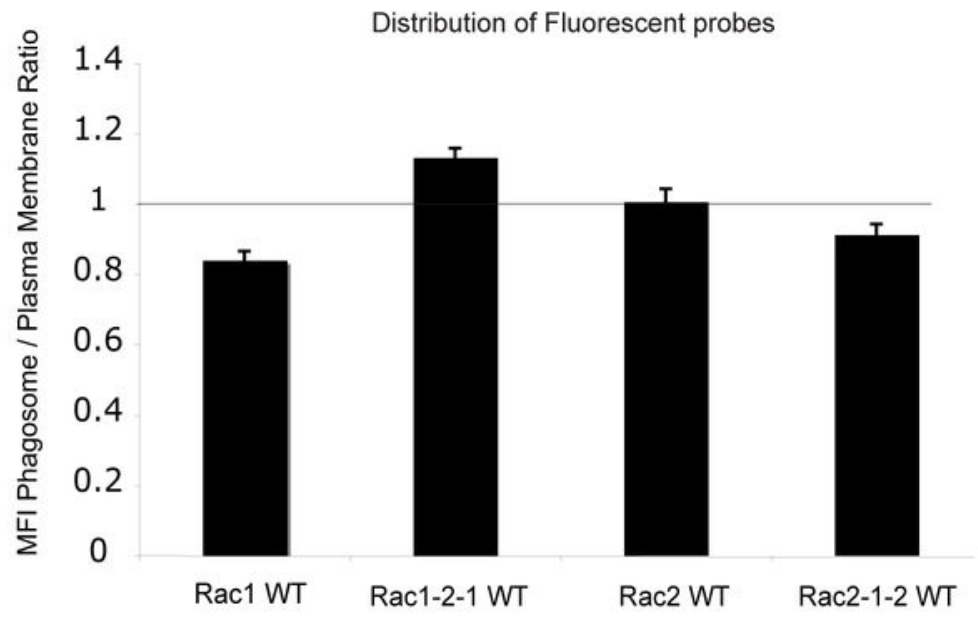

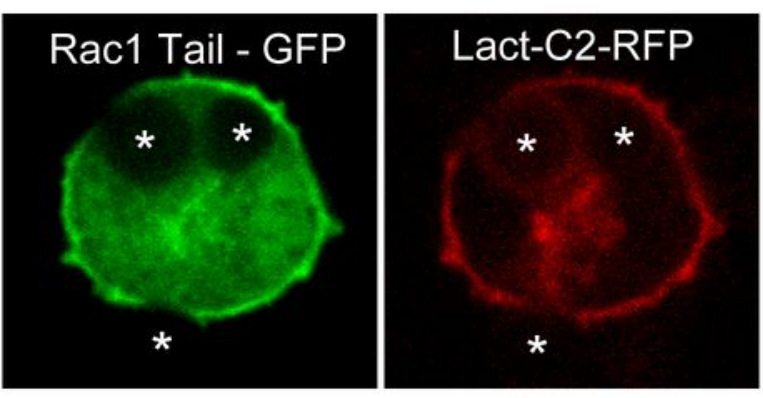


Figure 4
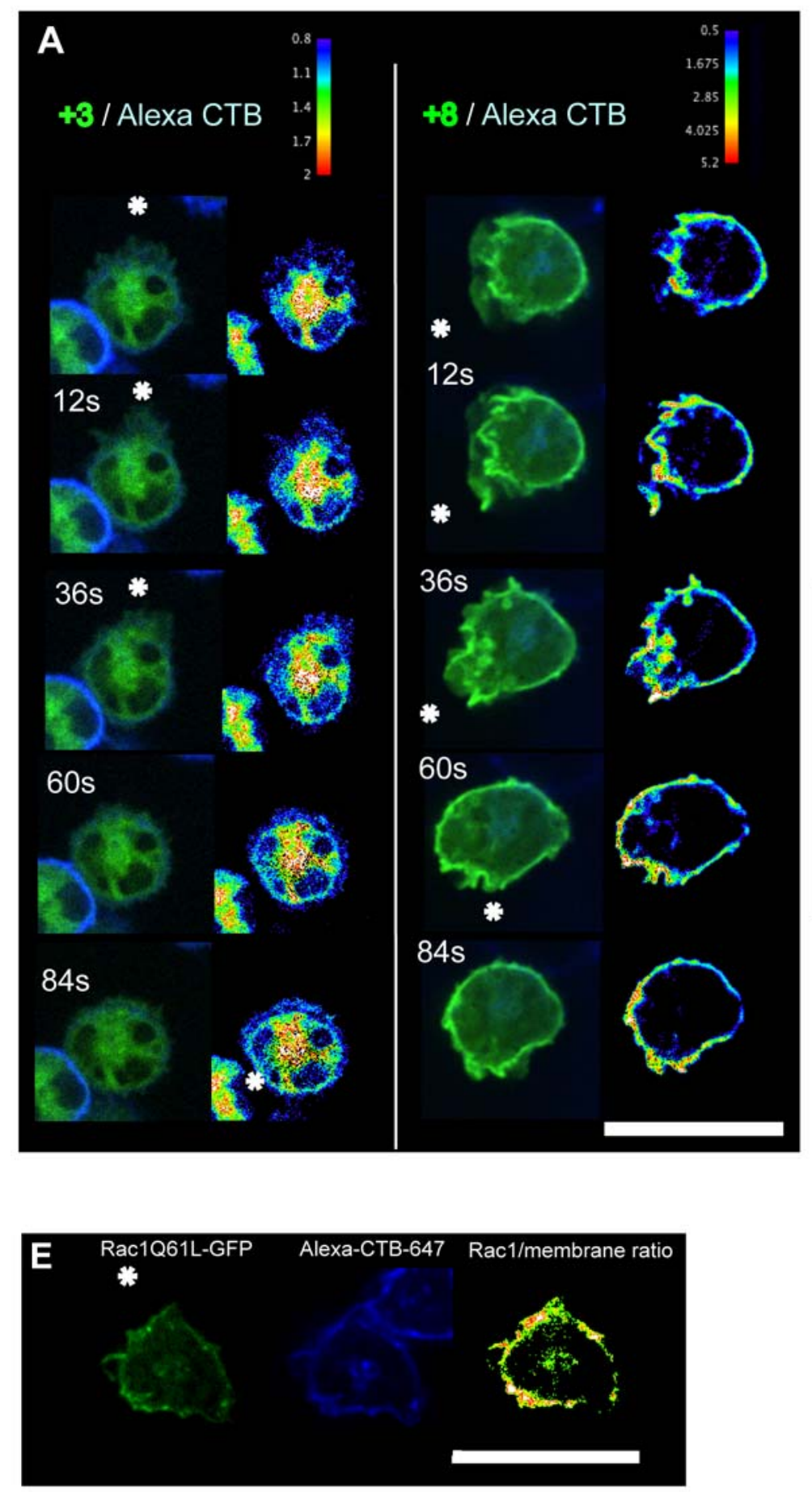
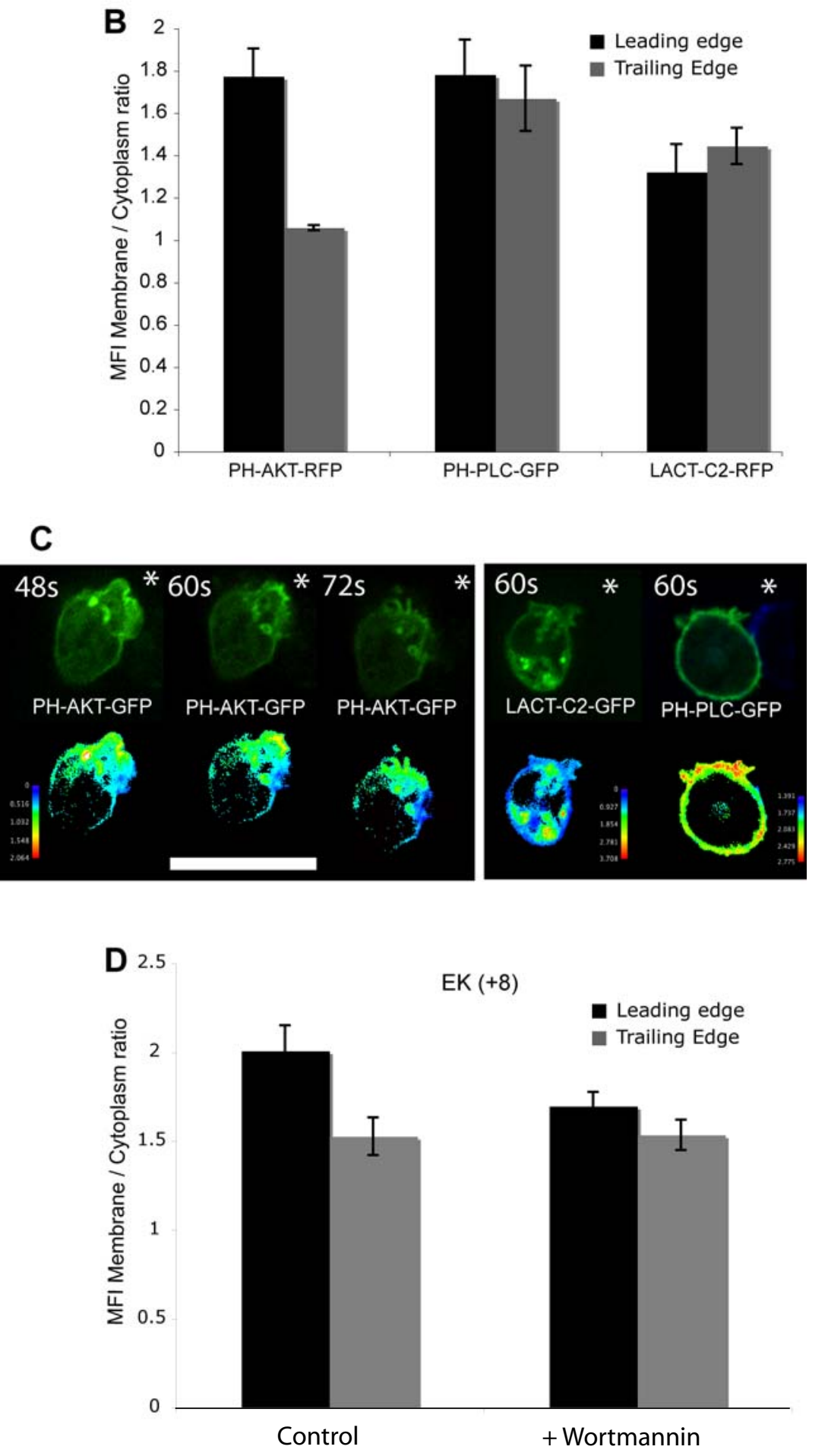
Figure 5

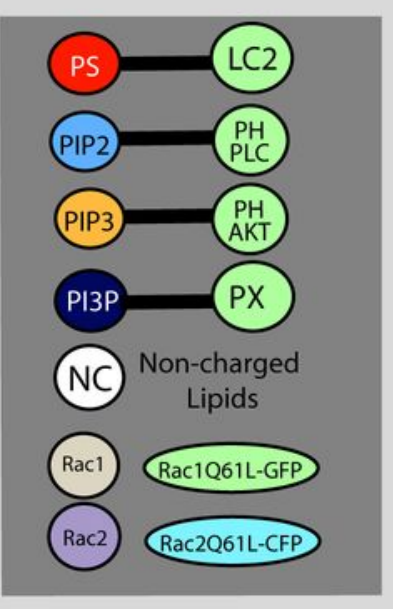

\section{(A) Resting}

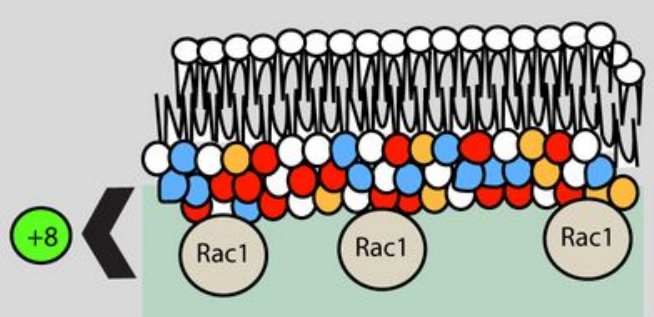

(B) Contact

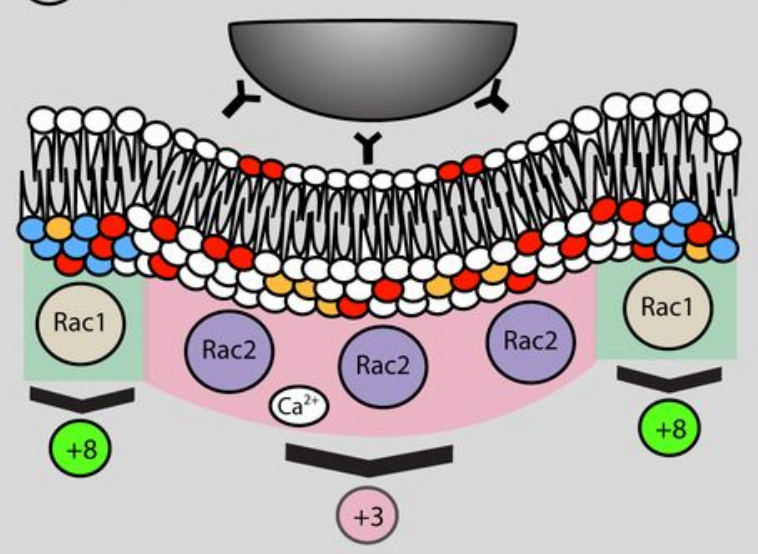

(C) Cup Phase

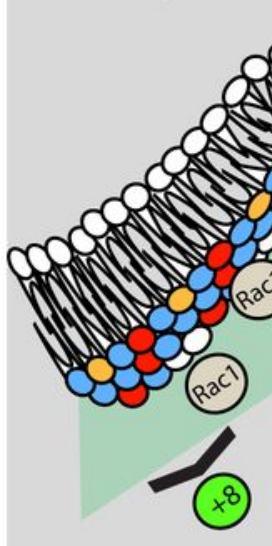

Chemotaxis (E)
(D) Cup Phase

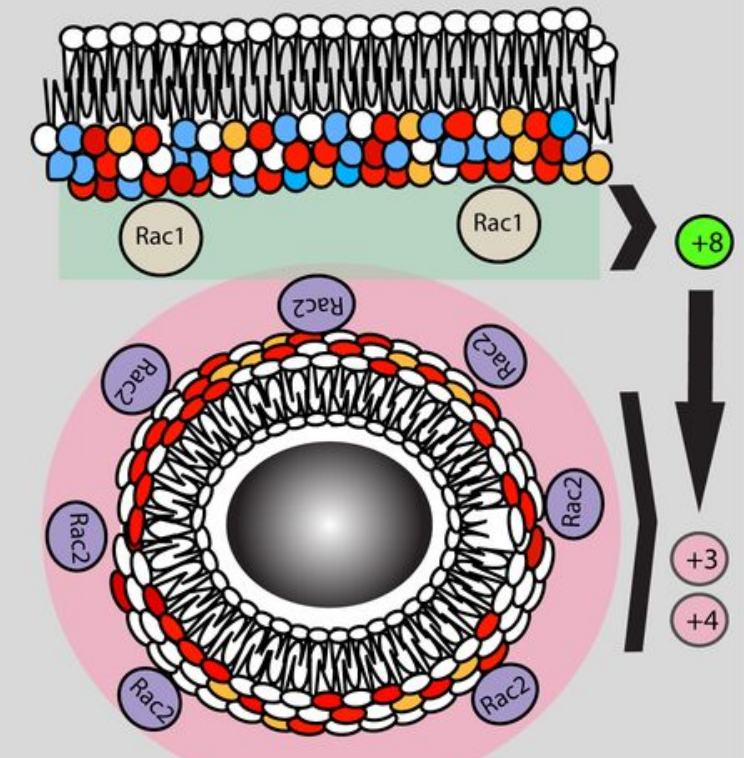

\section{(3)}

(x)

(4) $(44$
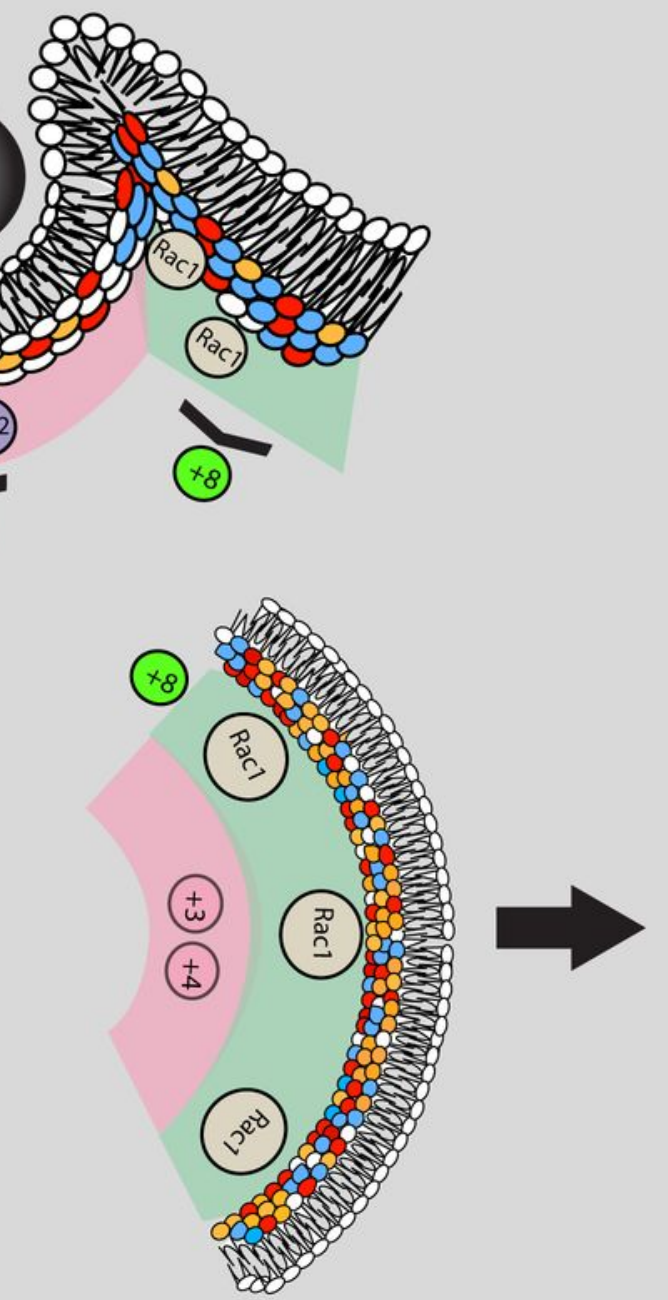